\title{
Assessment of bowel vascularity using indocyanine green fluorescence in incarcerated hernias
}

\author{
Abhishek Ganguly, Abhijith Acharya*, Manohar V. Pai, Alfred J. Augustine
}

\begin{abstract}
Department of General Surgery, Kasturba Medical College, Mangalore, Manipal Academy of Higher Education, Manipal, Karnataka, India
\end{abstract}

Received: 29 June 2021

Accepted: 11 August 2021

\author{
*Correspondence: \\ Dr. Abhijith Acharya, \\ E-mail: abh7588@gmail.com
}

Copyright: (C) the author(s), publisher and licensee Medip Academy. This is an open-access article distributed under the terms of the Creative Commons Attribution Non-Commercial License, which permits unrestricted non-commercial use, distribution, and reproduction in any medium, provided the original work is properly cited.

\begin{abstract}
Intra-operative assessment of bowel vascularity is often needed during emergent surgery. An objective test to determine the viability could reduce postoperative ischemic complications. Indocyanine green dye has been used for assessing bowel vascularity in colorectal surgery. The following study involving two patients demonstrates its use for assessing the viability of bowel in obstructed hernias after intraoperative intravenous injection. Stryker 1588 AIM laparoscopic setup was used for intraoperative fluorescence. In both cases, dusky areas were noted over the obstructed bowel segment on visual inspection. However, satisfactory fluorescence was seen after dye injection, and hence mesh repair was done without resecting the bowel. All the patients had a good post-operative recovery. Indocyanine green fluorescence may be a good modality to intraoperatively assess bowel vascularity.
\end{abstract}

Keywords: Obstructed hernias, Indocyanine green, Bowel vascularity

\section{INTRODUCTION}

Incarcerated inguinal hernia is one in which the content has become irreducible due to a narrow sac or due to adhesions in the sac. It presents with pain and irreducible swelling at the hernial site with features of intestinal obstruction.

It usually requires urgent exploration to release the incarcerated contents and to determine bowel viability. Visual assessment of intestinal viability can be unreliable (low predictive value) and operating surgeons may have different opinions. ${ }^{1}$

Many methods have been used like pulse oximetry, Doppler ultrasound, radioisotopes, hydrogen gas clearance, etc. with sensitivity above $80 \%$ but they have been found unsatisfactory and cumbersome. ${ }^{2}$

Ever since the index use of Indocyanine green (ICG) in laparoscopic cholecystectomy, it has been used in colorectal surgery and to detect sentinel lymph nodes in breast and gastric cancers. ICG enhanced fluorescence imaging is done after injection into a central or peripheral vein.

It provides a real-time assessment of bowel perfusion as it remains confined to the intravascular compartment. The following study involved the use of this dye intraoperatively for two patients with an obstructed hernia to check for bowel viability. This prospective single-center case series involved two consecutive inpatients with incarcerated hernias in the period from November 2019 to May 2020. Informed consent and institutional ethics committee approval was obtained. For both cases, an initial visual assessment of the bowel was done by two surgeons intraoperatively. Then ICG dye was used (25 $\mathrm{mg} / 10 \mathrm{ml}$ ). Stryker 1588 AIM laparoscopic setup was used for intraoperative fluorescence after injection of $2 \mathrm{ml}(5$ $\mathrm{mg}$ ) intravenously and $10 \mathrm{ml}$ normal saline flush.

Fluorescence was observed within sixty seconds of injection and darker areas signified reduced fluorescence. 


\section{CASE REPORT}

\section{Case 1}

A 78-years-old man, with no comorbidities, presented with right-sided groin swelling and pain for 2 days. He had vomited twice and had not passed flatus or stools. On examination, he had a $6 \times 5 \mathrm{~cm}$ firm swelling at the right groin which was tender on palpation. There was no cough impulse. Ultrasound confirmed the right inguinal hernia with small bowel loops and mesenteric fat (defect $2.7 \mathrm{~cm}$ ). A diagnosis of incarcerated hernia was made and the patient prepared for surgery. An open exploration was done. The muscles were divided and the sac was opened. Small bowel loops were found, dusky in color and edematous (Figure 1). The constriction ring was released. $5 \mathrm{mg}$ of ICG was given through a peripheral vein and fluorescence was assessed with the help of the Stryker 1588 AIM camera. As uniform fluorescence was observed, (Figure 2) the bowel was returned to the abdominal cavity and standard onlay mesh repair was done. Post-operatively patient recovered well, passed flatus on the second day, was started on orals, and discharged on the fourth day.

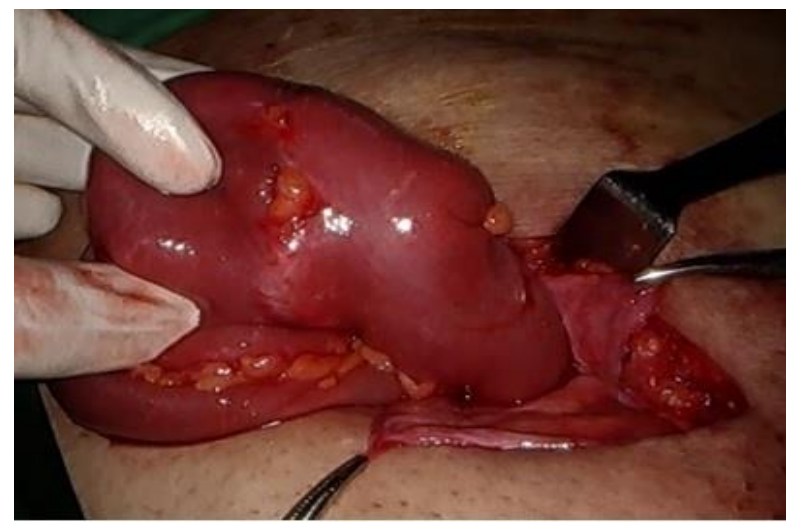

Figure 1: Obstructed small bowel loop.

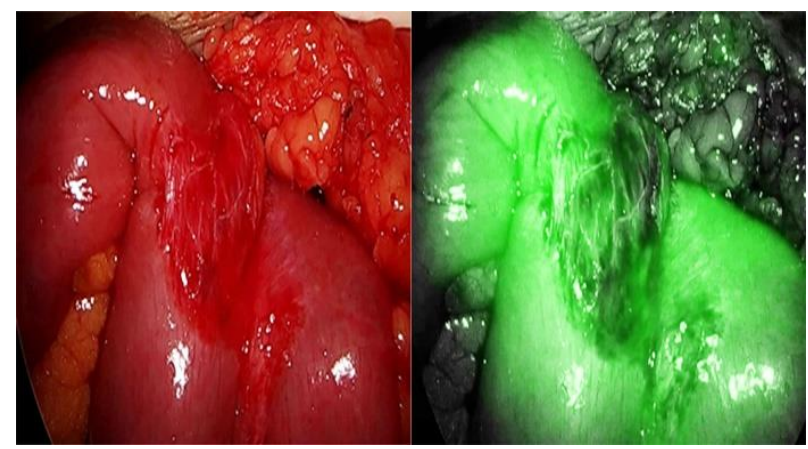

Figure 2: Small bowel loop before and after fluorescence.

\section{Case 2}

A 67-years-old male with no comorbidities presented with complaints of abdominal distension and bilious vomiting for 2 days. He had not passed stools for 1 day. On examination, he was conscious, alert, afebrile. An illdefined tender mass was palpated in the left lower abdomen below the umbilicus.

Ultrasound abdomen revealed mildly dilated bowel loops in the left iliac area and left lumbar hernia with bowel and omentum as content. Erect X-ray abdomen showed dilated bowel loops. After resuscitation with IV fluids, nasogastric aspiration, he underwent a CECT abdomen which showed an obstructed left Spigelian hernia (Figure 3). Open exploration was done. Intraoperatively, this was a Spigelian hernia with small bowel and sigmoid colon as content and both loops were edematous with a deep red color (Figure 4). When viewed under NIR mode after administration of intravenous ICG, good fluorescence was observed (Figure 5). A preperitoneal mesh repair was done after reducing the bowel. The patient passed flatus on the third day and tolerated oral feeds from day three onwards. He was discharged on day eight with no complications.

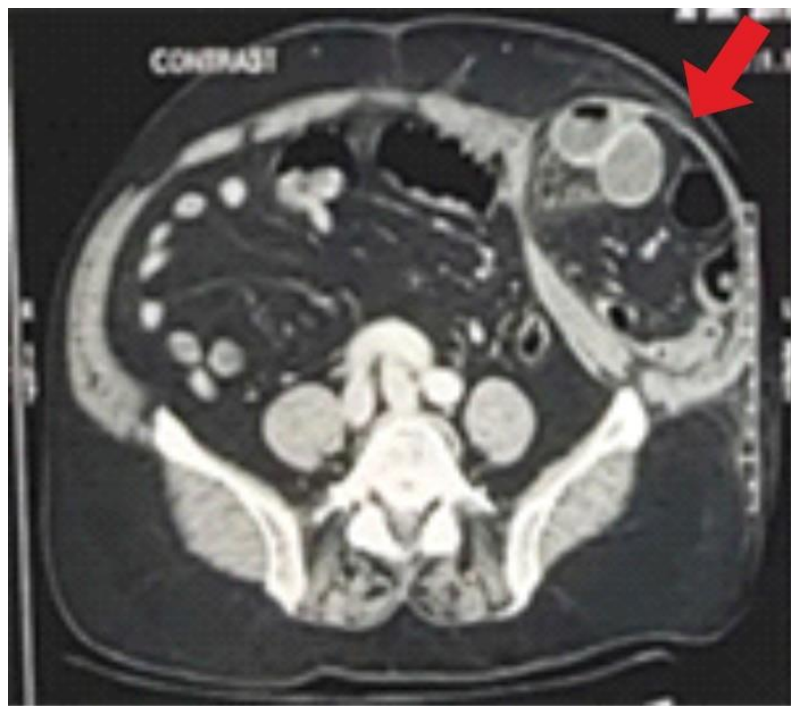

Figure 3: Contrast CT abdomen, pelvis- red arrow showing left spigelian hernia with obstructed bowel loops.

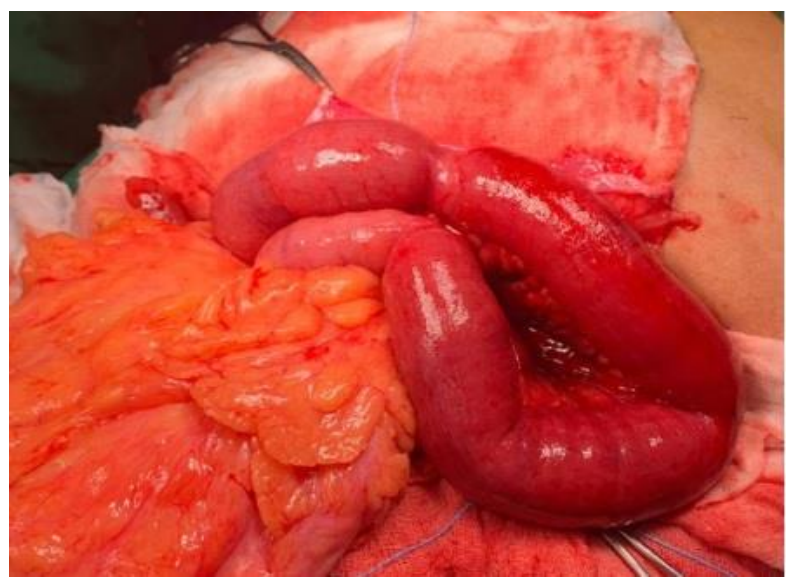

Figure 4: Intra-operative image showing obstructed bowel loops. 


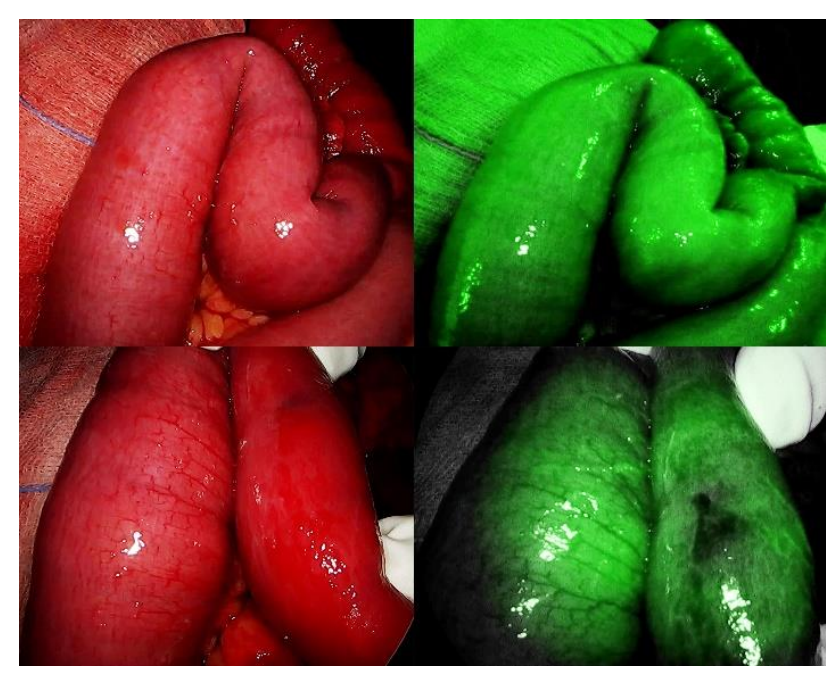

Figure 5: Bowel loops before and after ICG fluorescence.

\section{DISCUSSION}

This series involved 2 patients with incarcerated hernias. Incarcerated hernias represent a surgical emergency with a mortality of around $3 \% .^{3}$ Early surgical exploration and urgent release of the constriction ring are required. In a case where incarcerated bowel forms the content of the hernia, then assessment of bowel perfusion is of paramount importance. Clinical assessment of bowel viability, traditionally followed by most surgeons, relies on bowel color, presence of peristalsis, and pulsations in the mesenteric vessels. ${ }^{2}$ This has been proved to be highly subjective. The resection of viable bowel (judged ischemic by the surgeon), as well as conservative management of ischemic bowel (judged normal), can be disastrous for the patient. In cases with bowel resection, mortality may rise to $20 \%$, especially in elderly patients. Overall morbidity maybe around $20 \%$ post-emergency hernia surgery. ${ }^{3}$ To avoid these complications, an objective assessment of the bowel is necessary. Though there are several methods to assess intraoperative blood flow, many of these are either too expensive or either not practicable in the operating room.

ICG is a water-soluble dye eliminated by biliary excretion. After intravenous injection of the dye, it stays intravascular due to its protein binding capacity, and the fluorescence emission of the dye is detected by an infrared charge-coupled device (CCD) camera system. In our series, Stryker AIM 1588 laparoscopic setup was used for this purpose. Due to its short half-life and good safety profile, the dye can be used multiple times intraoperatively if needed and also in patients with renal impairment.

In both patients, after reduction of the incarcerated small bowel, the bowel was edematous and had a deep red color. Peristalsis could not be assessed. However, on administration of ICG, the bowel segment showed good fluorescence when viewed under NIR light. There was no difference of opinion among surgeons about vascularity.
Therefore, a confident decision could be made to avoid resection and anastomosis. The patients had an uneventful clinical recovery and none of them had adverse effects after ICG injection.

At most centers, the need for resection in incarcerated hernias is usually decided by the surgeons based on their clinical judgment. The intraoperative use of ICG was unique in our report as it could pave way for a more objective assessment of bowel viability. The possible limitation in this technique would be acquiring the expensive setup for assessing the fluorescence. No significant toxic effects have been seen in humans with doses up to $5 \mathrm{mg} / \mathrm{kg}$ body weight. ${ }^{4}$ False-positive results with this dye are unlikely.

Despite several reports advocating the laparoscopic approach in emergency hernia repair, its role in acute settings is not well established yet. ${ }^{5}$ Hence open exploration was done in all our cases. As per the 2017 update of World Society of Emergency Surgery (WSES) guidelines, ${ }^{5}$ prosthetic mesh was used for repair of hernia in both cases after reducing the contents.

There has been a paucity of studies exploring the use of ICG in incarcerated hernias. Ryu et al used ICG to assess bowel perfusion in obstructed umbilical, femoral, and inguinal hernias. ${ }^{6-8}$ In these three cases bowel showed deep red discoloration on gross appearance but good fluorescence was observed with ICG. Hence, resection and anastomosis were avoided. Similarly, Daskopolou et al. presented a case report of a 92 years old woman with strangulated obturator hernia. Here, NIR light with ICG not only helped with visualization of nonviable bowel but also identified healthy bowel ends for anastomosis. ${ }^{9}$ In a case report by Michael OSSEIS et al, ICG imaging identified the limits between ischemic and vascularised bowel. ${ }^{10}$

\section{CONCLUSION}

Very few reports have assessed the use of ICG for bowel viability in hernia surgeries. Due to its excellent safety profile and objective assessment, ICG fluorescence seems to be a promising technique in cases of incarcerated hernias. The need for further studies with a larger sample size may be emphasized.

\section{Funding: No funding sources \\ Conflict of interest: None declared \\ Ethical approval: Not required}

\section{REFERENCES}

1. Horgan PG, Gorey TF. Operative assessment of intestinal viability. Surg Clin North Am. 1992;72(1):143-55.

2. Urbanavicius L, Pattyn P, Putte DV, Venskutonis D. How to assess intestinal viability during surgery: A 
review of techniques. World J Gastrointest Surg. 2011;3(5):59-69.

3. Ceresoli M, Carissimi F, Nigro A, Fransvea P, Lepre L, Braga M, et al. Emergency hernia repair in the elderly: multivariate analysis of morbidity and mortality from an Italian registry. Hernia. 2020.

4. Alander JT, Kaartinen I, Laakso A, Patila T, Spillmann T, Tuchin VV, et al. A review of indocyanine green fluorescent imaging in surgery. Int J Biomed Imaging. 2012;940585.

5. Birindelli A, Sartelli M, Saverio S, Coccolini F, Ansaloni L, Ramshorst GH, et al. 2017 update of the WSES guidelines for emergency repair of complicated abdominal wall hernias. World J Emerg Surg. 2017;12:37.

6. Ryu S, Yoshida M, Ohdaira H, Tsutsui N, Suzuki N, Ito $\mathrm{E}$, et al. A case of incarcerated femoral hernia with intestinal blood flow assessment by brightfield fullcolor near-infrared fluorescence camera: Report of a case. Int J Surg Case Rep. 2016;29:234-6.

7. Ryu S, Yoshida M, Ohdaira H, Tsutsui N, Suzuki N, Ito E, et al. Blood flow evaluation using PINPOINT® in a case of incarcerated inguinal hernia: A case report. Asian J Endosc Surg. 2017;10(1):75-8.
8. Ryu S, Yoshida M, Ohdaira H, Tsutsui N, Suzuki N, Ito $\mathrm{E}$, et al. Intestinal blood flow assessment by indocyanine green fluorescence imaging in a patient with the incarcerated umbilical hernia: Report of a case. Ann Med Surg. 2016;8(13):40-2.

9. Daskalopoulou D, Kankam J, Plambeck J, Ambe PC, Zarras K. Intraoperative real-time fluorescence angiography with indocyanine green for evaluation of intestinal viability during surgery for an incarcerated obturator hernia: a case report. Patient Saf Surg. 2018;12:24.

10. Osseis M, Lim C, Moussallem T, Osseis M, Lahat E, Salloum C, et al. Indocyanine green-fluorescence imaging in the assessment of intestinal viability during emergency bowel surgery. J Med Liban. 2018;66(5):279-83.

Cite this article as: Ganguly A, Acharya A, Pai MV, Augustine AJ. Assessment of bowel vascularity using indocyanine green fluorescence in incarcerated hernias. Int Surg J 2021;8:2834-7. 